7. Dutton-Regester K, Kakavand H, Aoude LG, et al. Melanomas of un known primary have a mutation profile consistent with cutaneous sunexposed melanoma. Pigment Cell Melanoma Res 2013; 26: 852-60.

8. Newell F, Kong Y, Wilmott JS, et al. Whole-genome landscape of mucosal melanoma reveals diverse drivers and therapeutic targets. Nat Commun 2019; 10: 3163.

9. Hintzsche JD, Gorden NT, Amato CM, et al. Whole-exome sequencing identifies recurrent SF3B1 R625 mutation and comutation of NF1 and KIT in mucosal melanoma. Melanoma Res 2017; 27: 189-99.

10. Yang C, Sanchez-Vega F, Chang JC, et al. Lung-only melanoma: UV mutational signature supports origin from occult cutaneous primaries and argues against the concept of primary pulmonary melanoma. Mod Pathol 2020; 33: 2244-55.

11. Hutchinson KE, Lipson D, Stephens PJ, et al. BRAF fusions define a distinct molecular subset of melanomas with potential sensitivity to MEK inhibition. Clin Cancer Res 2013; 19: 6696-702.

12. Krumbholz M, Jung R, Bradtke J, Reinhardt D, Stachel D, Metzler M Response monitoring of infant acute myeloid leukemia treatment by quantification of the tumor specific MLL-FNBP1 fusion gene. Leuk Lymphoma 2015; 56: 793-6.

DOI: https://doi.org/10.1016/j.pathol.2020.12.009

\section{Sialadenoma papilliferum of the bronchus: focus on histological and genetic distinction from mixed squamous cell and glandular papilloma}

Sir,

Sialadenoma papilliferum (SP) is a rare salivary gland tumour consisting of both an exophytic projection of squamous epithelium and an inverted papillary proliferation of glandular epithelium. ${ }^{1}$ SP usually arises in the intraoral minor salivary glands and rarely occurs in the bronchus. To our knowledge, there are four English case reports on SP of the bronchus or trachea. ${ }^{2-5}$ However, some tumours designated as bronchial SPs in previous publications appeared to represent mixed squamous cell and glandular papilloma (MP); judging from published illustrations, endobronchial prominent papillary structure with a broad fibrovascular core, that is characteristic for MP, was observed in some tumours. Conversely, in a recent publication, a bronchial tumour showing SP-like morphology was described as MP. ${ }^{6}$ No publications in the relevant literature have focused on the histological distinction between SP and MP. We herein report a case of SP of the bronchus and discuss the histological and genetic distinction between SP and MP.

The patient was a 63-year-old man with a history of coughing. Bronchoscopy revealed a whitish, lobulated lesion protruding in the lumen of the left main bronchus (Fig. 1A,B). The patient had undergone endoscopic snare tumourectomy following a diagnosis of benign papilloma, likely an MP, by a pathological examination of a biopsy specimen at a previous institute.

Histologically, a papillary squamous surface and a contiguously endophytic ductal proliferation was seen (Fig. 1C). Oncocytic metaplasia was not found. There was no cytologic atypia or necrosis. Immunohistochemically, p63 [4A4, ready-to-use (RTU); Nichirei Biosciences, Tokyo, Japan] was positive in the surface squamous epithelium and basal layer of the ductal component, while SOX10 (BC34, RTU; Nichirei Biosciences, Japan) was positive in the ductal component (Fig. 1D,E). S100 (polyclonal, RTU; Dako, Australia) was focally positive in the ductal component. Overall, the histological and immunohistochemical findings were consistent with SP.

Recurrent oncogenic alterations in $B R A F$, followed by $H R A S$, have been reported in SP of the intraoral minor salivary glands, ${ }^{7,8}$ but a mutation analysis for SP of the bronchus has not been reported. We thus extracted DNA from a formalin fixed, paraffin embedded specimen and performed targeted sequencing using the Ion AmpliSeq custom panel (Thermo Fisher Scientific, USA) for 23 cancer related genes, as described in detail elsewhere. ${ }^{9}$ No putative driver mutations, including $B R A F, H R A S$ and $A K T 1$ mutations, were detected at a mean depth of $\times 3574$. The lack of $B R A F \mathrm{~V} 600 \mathrm{E}$ mutations was also confirmed by immunohistochemistry using BRAF V600E mutation specific antibody (VE1, RTU; Ventana Medical Systems, USA). These genetic results did not appear inconsistent with the histological classification of the present case as SP; indeed, a lack of BRAF and HRAS mutations has been reported in $20 \%$ of SP cases.

No tumour recurrence was found for 9 months after the last follow-up bronchoscopy following tumour resection.

The major differential diagnosis of bronchial SP is MP. Intrabronchial papillary growth and constituent cells of squamous and glandular cells with a layer of basal cells are findings common to SP and MP (Fig. 2A). However, the distribution of squamous and ductal components is arranged with regularity in SP, while the constituent cells are mixed together in MP. Furthermore, ciliated cells are usually intermixed within MP. However, the distinction between SP and MP may be difficult in small biopsy specimens, and an immunohistochemical analysis may need to be conducted to make a correct diagnosis. Consistent with a previous study, in the present case, SOX10 was positive in the ductal component of SP (Fig. 1E). In addition, due to the lack of published studies on SOX10 staining in MP, we examined the SOX10 immunohistochemistry for four MPs in a recently published cohort. ${ }^{10}$ SOX10 was negative in every epithelial component in all four MPs (Fig. 2B), while SOX10 was positive for bronchial glands as an internal control (Fig. 2C,D).

SOX10 is positive for the acini and intercalated ducts of the salivary glands as well as for the tumours derived from these cells. ${ }^{11}$ Therefore, SOX10 positivity in the ductal component of SP may suggest that the component is derived from the intercalated duct of the minor salivary gland and bronchial gland. ${ }^{7}$ In contrast, MP arises not only in the bronchi, but also in the bronchiole without a submucosal bronchial gland. ${ }^{10,12}$ Taken together, these findings along with the SOX10 negativity in MP suggest that MP derives from the surface respiratory epithelium.

S100 is another marker for the ductal component of SP. However, S100 appeared to exhibit lower sensitivity for the ductal component of SP than SOX10. Consistent with previous studies, ${ }^{3,7}$ the ductal component in the present case of SP showed diffuse and robust positivity for SOX10 but focal positivity for S100. Additionally, we examined the S100 immunohistochemistry for four MPs. S100 was negative in every epithelial component of MPs, while variable numbers of S100-positive dendritic cells were intermixed within the papillomas. These dendritic cells were negative for SOX10. Therefore, these findings suggest that SOX10 could be a 

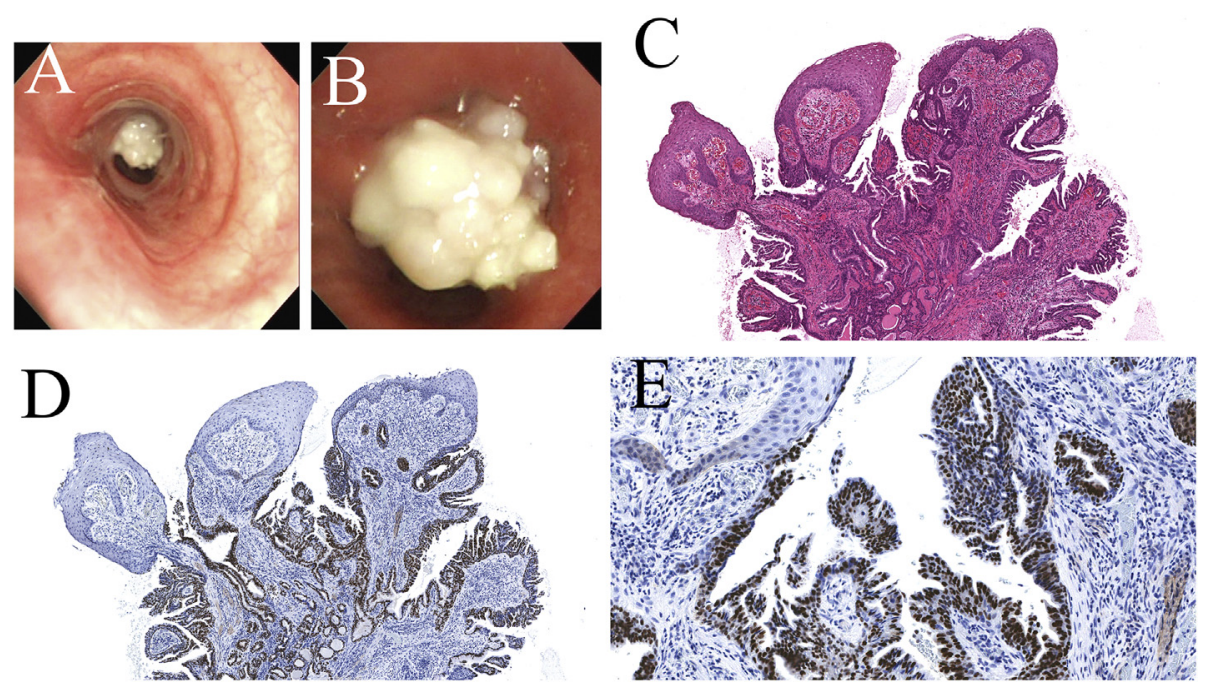

Fig. 1 Gross, microscopic, and immunohistochemical findings of a sialadenoma papilliferum of the bronchus. (A) An endoscopic examination revealed a protruding tumour in the left main bronchus. (B) The tumour was a whitish lobulated lesion, measuring around 10 mm. (C) The tumour showed an exophytic papillary squamous proliferation contiguous with an endophytic ductal infolding. (D) Under a low-power view, the endophytic ductal component showed positive staining for SOX10. (E) Under a high-power view, both abluminal cells and basal cells were positive for SOX10.
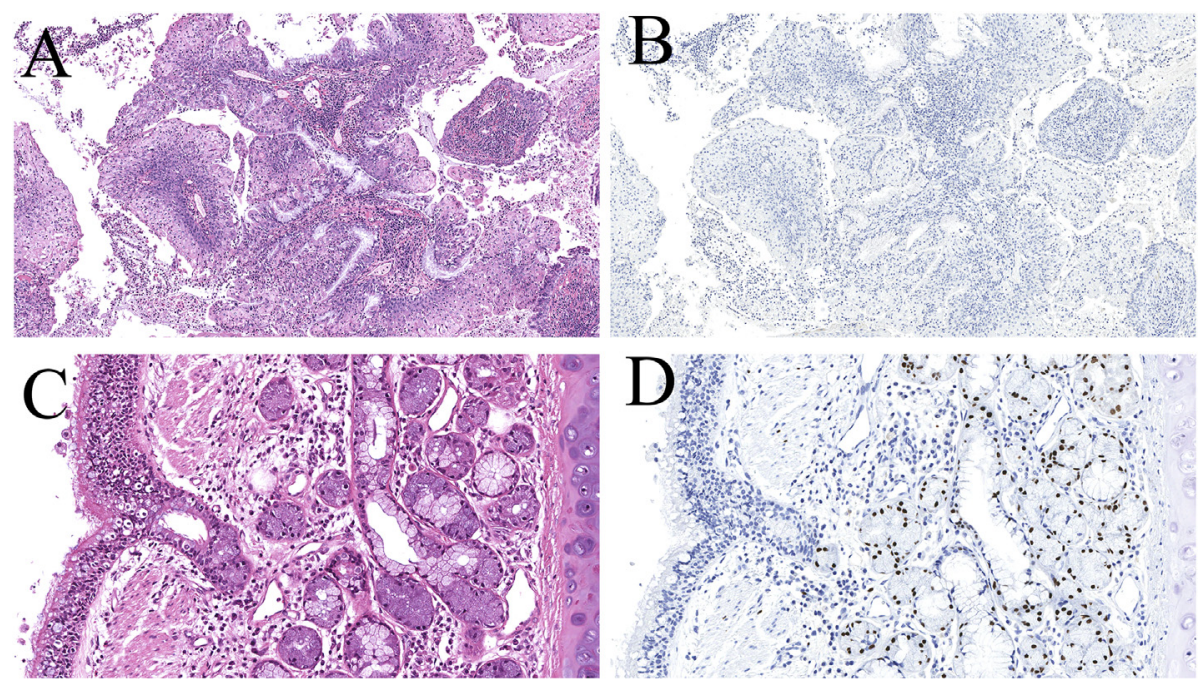

Fig. 2 Microscopic and immunohistochemical findings of mixed squamous cell and glandular papilloma (A,B) and bronchial gland (C,D). (A) Glandular cells and squamous cells are mixed up together within the papilloma. (B) The tumour was completely negative for SOX10. (C,D) The submucosal bronchial gland, not surface bronchial epithelium, showed positive staining for SOX10: (C) H\&E staining; (D) SOX10.

Table 1 Comparison between SP and MP

\begin{tabular}{|c|c|c|}
\hline Anatomical site in the lung & Bronchus & Bronchus and bronchiole \\
\hline Gross findings & Polypoid & Polypoid \\
\hline Putative histological origin & Minor salivary gland, bronchial gland & Surface bronchial epithelium \\
\hline Constituent cells & Squamous cells, and ductal cells with basal cells & $\begin{array}{l}\text { Squamous cells, glandular cells, ciliated cells, and basal } \\
\text { cells }\end{array}$ \\
\hline Distribution of constituent cells & $\begin{array}{l}\text { Exophytic projection of squamous epithelium and an } \\
\text { inverted papillary proliferation of ductal epithelium }\end{array}$ & $\begin{array}{l}\text { Intermingled distribution of squamous, glandular, and } \\
\text { ciliated cells }\end{array}$ \\
\hline SOX10 immunohistochemistry & $\begin{array}{l}\text { Positive in the ductal component but negative in the } \\
\text { squamous component }\end{array}$ & Negative in every epithelial component \\
\hline Genetic alteration & $\begin{array}{l}B R A F \text { mutations }(70 \%) \text {, followed by } H R A S \text { mutations } \\
(10 \%) \text { or the lack of mutations }(20 \%)^{8}\end{array}$ & $\begin{array}{l}A K T 1 \text { mutations concurrent with } B R A F(75 \%) \text { or } H R A S \\
(25 \%) \text { mutations }{ }^{10}\end{array}$ \\
\hline
\end{tabular}

MP, mixed squamous cell and glandular papilloma; SP, sialadenoma papilliferum. 
superior marker to $\mathrm{S} 100$ in the setting of differentiation between SP and MP.

Recent studies on genetic analyses for SP and MP have shown that these two tumours are genetically different; $B R A F$ or HRAS mutations have been detected in both SP and MP, ${ }^{7,8,10}$ but concurrent $A K T 1$ mutations are found in $\mathrm{MP}^{10}$ and are absent in SP. ${ }^{8}$ Indeed, $A K T 1$ mutations were not detected in the present case of SP. Further studies are warranted for the genetic characterisation of SP arising in unusual anatomical sites, including the bronchus.

Recently, Hsieh, et al. reported that SP consists of two morphologically distinct subtypes: conventional subtype and oncocytic variant. ${ }^{7}$ Based on SOX10 positivity and the lack of oncocytic change, the present case of SP would be classified as the conventional subtype. In oncocytic SP, the ductal component is entirely lined by SOX10-negative oncocytic cells with abundant eosinophilic cytoplasm and blandlooking nuclei. ${ }^{7}$ Among the constituent cells of MP, ciliated cells may occasionally show eosinophilic cytoplasm. ${ }^{10}$ Therefore, the distribution and cytomorphology of oncocytic cells are different between MP and oncocytic SP, although it remains unknown whether oncocytic SP can occur in the bronchus.

We summarised a comparison of the clinicopathological characteristics between SP and MP in Table 1. These two tumours are entirely different entities in terms of their origin. The differing histological findings along with the results of SOX10 immunohistochemistry can help discriminate between SP and MP.

Conflicts of interest and sources of funding: The authors state that there are no conflicts of interest to disclose.

\section{Eiichi Sasaki $^{1}$, Akari Iwakoshi ${ }^{1,2}$, Katsuhiro Masago ${ }^{1}$, Nobuhiro Hanai ${ }^{3}$, Masahide Oki ${ }^{4}$}

${ }^{1}$ Department of Pathology and Molecular Diagnostics, Aichi Cancer Center Hospital, Nagoya, Japan; ${ }^{2}$ Department of Pathology, Nagoya Medical Center, Nagoya, Japan; ${ }^{3}$ Department of Head and Neck Surgery, Aichi Cancer Center Hospital, Nagoya, Japan; ${ }^{4}$ Department of Respiratory Medicine, Nagoya Medical Center, Nagoya, Japan

Contact Dr Eiichi Sasaki.

E-mail: esasaki@aichi-cc.jp

1. Foschini MP, Bell D, Katabi N. Sialadenoma papilliferum. In: El Naggar AK, Chan JKC, Grandis JR, Takata T, Slootweg PJ, editors. WHO Classification of Head and Neck Tumours. Lyon: IARC Press, 2017; 192.

2. Grzesik P, Passik C, Mahfoozi A. An unusual cause of dyspnea. Ann Thorac Surg 2016; 102: e333-4.

3. Honda Y, Shiraishi K, Nomori H, et al. Sialadenoma papilliferum of the bronchus: rare tracheobronchial tumor of salivary gland type. Pathol Int 2009; 59: 572-6.

4. Bobos M, Hytiroglou P, Karkavelas G, et al. Sialadenoma papilliferum of bronchus. Virchows Arch 2003; 443: 695-9.

5. Campisi A, Dell'Amore A, Bertolaccini L, et al. Sialadenoma papilliferum of the bronchus: a rare tumour of salivary gland origin. $A d v$ Respir Med 2020; 88: 267-70.

6. Travis W, Nicholson A, Geisinger K, Brambilla E. AFIP Atlas of Tumor Pathology, Series 4: Tumors of the Lower Respiratory Tract. Arlington: American Registry of Pathology, Armed Forces Institute of Pathology, 2019; Chapter 16, Papillomas.

7. Hsieh MS, Bishop JA, Wang YP, et al. Salivary sialadenoma papilliferum consists of two morphologically, immunophenotypically, and genetically distinct subtypes. Head Neck Pathol 2020; 14: 489-96.
8. Nakaguro M, Urano M, Ogawa I, et al. Histopathological evaluation of minor salivary gland papillary-cystic tumours: focus on genetic alterations in sialadenoma papilliferum and intraductal papillary mucinous neoplasm. Histopathology 2020; 76: 411-22.

9. Sasaki E, Masago K, Fujita S, Hanai N, Yatabe Y. Frequent KRAS and HRAS mutations in squamous cell papillomas of the head and neck. J Pathol Clin Res 2020; 6: 154-9.

10. Sasaki E, Masago K, Fujita S, et al. AKT1 mutations in peripheral bronchiolar papilloma: glandular papilloma and mixed squamous cell and glandular papilloma is distinct from bronchiolar adenoma. Am J Surg Pathol 2021; 1: 119-26.

11. Ohtomo R, Mori T, Shibata S, et al. SOX10 is a novel marker of acinus and intercalated duct differentiation in salivary gland tumors: a clue to the histogenesis for tumor diagnosis. Mod Pathol 2013; 26: 1041-50.

12. Flieder DB, Koss MN, Nicholson A, Sesterhenn IA, Petras RE, Travis WD. Solitary pulmonary papillomas in adults: a clinicopathologic and in situ hybridization study of 14 cases combined with 27 cases in the literature. Am J Surg Pathol 1998; 22: 1328-42.

DOI: https://doi.org/10.1016/j.pathol.2020.11.012

\section{Eosinophilic renal cell carcinoma with isolated MTOR mutation metastatic to the liver: a novel case}

Sir,

Although significant advancements have been made in classifying renal cell carcinoma (RCC), approximately 5$7 \%$ of cases are still characterised as 'unclassified' RCCs. Studies of these 'unclassified' cases have shown that $\sim 70-$ $80 \%$ exhibit eosinophilic cytoplasm and renal oncocytoma (RO) or chromophobe RCC (CHRCC) phenotypes. ${ }^{1}$ Molecular investigations have revealed that a subset of eosinophilic renal neoplasms with heterogeneous morphology and immunohistochemical (IHC) profiles harboured mutations in the TSC/MTOR pathway as their primary molecular alteration. $^{2-6}$ The metastatic potential of these neoplasms is still unknown and will require further identification of more cases and follow-up. Chen et al. identified two cases of MTOR-mutated RCCs that were both organ confined. ${ }^{3}$ In our previous study, we identified two cases of MTORmutated RCCs that were also both organ confined. ${ }^{5}$ In this article, we report the first case of a MTOR-mutated RCC that metastasised to the liver.

A 65-year-old male presented for consultation after having been diagnosed with metastatic RCC at an outside hospital. He had initially presented with low volume metastatic disease to the liver and lung and underwent a left radical nephrectomy that demonstrated an $8.5 \mathrm{~cm}$ renal mass. The lesion was diagnosed as unclassified RCC, Fuhrman grade 4 at the outside hospital. Following surgery, he was first treated with sunitinib with stable disease followed by nivolumab, which led to a partial response. Axitinib was then started followed by hepatic arterial ytrrium-90 microspheres for persistent liver only disease. His treatment course was complicated by shortness of breath after which he was diagnosed with immunotherapy related pneumonitis.

We received the consult slides from the original radical nephrectomy, which demonstrated an $8.5 \times 5.6 \times 5.5 \mathrm{~cm}$ wellcircumscribed lesion with extensive haemorrhage, necrosis, and calcifications (Fig. 1A). The tumour invaded into the renal sinus and perinephric adipose tissue, but the margins of resection (soft tissue, ureter, renal artery, and renal vein) were 